HUMANITAS ISayı - Number: 3

Bahar / Spring, Tekirdağ, 2014

\title{
TRIANGLE REMÉMORATIF : ISTANBUL - LE JEUNE PAMUK - ARTISTES/ÉCRIVAINS FRANÇAIS
}

\author{
Mümtaz KAYA ${ }^{1}$
}

Öz: Ístanbul, Hatıralar ve Şehir (2003) adlı yapıtında, anlatıcı-yazar Orhan Pamuk, genç Orhan'ın yaşamıyla özdeşleşen İstanbul hakkındaki anılarını kaleme alırken, Fransız gezgin, mimar ve ressam Antoine Ignace Melling'in (1763-1831) İstanbul'u konu alan gravürlerine yer verir ve her birine bir bölüm ayırdığı, 19. Yüzyıl Fransız yazın dünyasının önemli yazarları Théophile Gautier, Gérard de Nerval ve Gustave Flaubert'in İstanbul'a ilişkin yazdıklarını alt metin olarak kendi yapıtına taşır. Pamuk'un o yazarlarla iletişim halinde olmasının, okurları bu yazarları farklı bir bakış açısıyla okumaya davet etmesi kadar, kaleme almaya çalıştığı anılarını canlandırmak için başvurduğu bir yol olduğunu da düşünmekteyiz. Özyaşamöykülerinde kullanılan alt metinler, bu altmetinlerden hareketle oluşturulan anılar, özyaşamöykülerin doğruluğu konusunda okuru kuşkuya düşürse de, okur, anlatıc1-yazar ile kendisi arasında, gerçeklerin tüm çıplaklığıyla anlatılacağı konusunda "varılan anlaşmaya" (pacte autobiographique) inanmak durumundadır. Çalışmamızda, yapıtın Türkçe başlığını (İstanbul, Hatıralar ve Şehir) ve Fransızca başlığını (Istanbul, Souvenirs d'une ville) yaşamöyküsel ve özyaşamöyküsel göndermeler bağlamında karşılaştırdıktan sonra bu yapıtın, Philippe Lejeune'in 1975 yılında tanımını verdiği "Özyaşamöyküsel Anlaşma”ya (Le Pacte autobiographique) ve Özyaşamöyküsel ölçütlere ne kadar uyduğunu, okur ile varılan bir "içtenlik anlaşması" (Pacte de sincérité/franchise) olup olmadığını, "anlaşmaya" ne derecede uyulmaya çalışıldığını saptamaya çalışacağız. Öte yandan, yukarıda andığımız Fransız sanatçılarının alt metinlerinin, anıların oluşturulmasındaki önemi üzerinde de durulacaktır.

Anahtar Sözcükler: Orhan Pamuk, Istanbul, Souvenirs d'une ville, Autobiographie, Récit intime, Récit rétrospectif, Embrayeurs biographiques, Rencontres biographiques, Biographie urbaine, Melling, Baudelaire, Flaubert, Gautier, Nerval.

\section{Introduction et objectif}

Que ce soit sous forme de mémoires, journal intime, confessions, souvenirs, autoportrait, biographie, autobiographie ou encore roman autobiographique, les trois dernières décennies du XXème siècle et la première décennie de notre

\footnotetext{
${ }^{1}$ Doç. Dr., Bilkent Üniversitesi, Uygulamalı Yabancı Diller Yükekokulu. mumtaz@bilkent.edu.tr
} 
siècle témoignent de beaucoup d'auteurs ayant entrepris l'écriture intime, voire le biographique. Comme le souligne Michel Maillard, dans son ouvrage intitulé L'autobiographie et la biographie « on pourrait même parler d'inflation autobiographique pour évoquer l'extraordinaire développement qu'ont pu connaitre tous les récits intimistes ou exhibitionnistes d'une époque qui semble aimer dire et lire l'intimité » $(2001$, p. 8). Dû à une forte sollicitation de la part des lecteurs, ce genre intimiste est devenu une des plus importantes composantes de la littérature française et un grand nombre d'écrivains très connus comme André Gide, Jean-Paul Sartre, Simone de Beauvoir, Marguerite Duras, Jorge Semprun, Annie Ernaux ou Nathalie Sarraute s'y sont prêtés avec beaucoup de succès.

Lauréat du prix Nobel de littérature en 2006, Orhan Pamuk semble ne pas échapper à ce genre intimiste. Son œuvre Istanbul, Souvenirs d'une ville publiée en 2003 (2007, pour la traduction française) ${ }^{2}$ en est un bel exemple. En effet, l'œuvre débute par un incipit relatant la plus petite enfance d'Orhan Pamuk qui a 50 ans quand il entreprend ce travail- et se termine à l'époque où celui-ci a 22 ans. A travers ses souvenirs d'enfance et ceux d'Istanbul, l'auteur est à la recherche de son identité dans le « hüzün » de sa ville qui est « un sentiment noir éprouvé conjointement par İstanbul et par ses millions d'habitants » (2007, p. 141). De ce fait, le « hüzün » qui est un sentiment mêlé de perte, de souffrance, de misère, de nostalgie, voire cette tristesse profonde " qui se distingue bien du sentiment de mélancolie qui renvoie à l'état mental d'une seule personne »(2007, p. 141) est le fil conducteur du récit. Ainsi, en s'identifiant à l'état d'âme d'İstanbul et de ses habitants, l'auteur essaie de cristalliser ses souvenirs: il raconte l'histoire de son « double »-celle dont il essaie de se remémorer avec le support des photographies en noir et blanc dont notamment des gravures d' Antoine Ignace Melling - à travers la double histoire d'İstanbul, celle qui est gravée dans la mémoire du jeune Orhan Pamuk, et celle qu'il reconstitue notamment grâce aux récits des écrivains voyageurs français qui ont décrit Istanbul un siècle avant sa naissance.

Dans le cadre de ce triangle remémoratif, l'objectif de ce travail sera d'une part, essayer de retrouver à partir du titre et à travers la narration, les éléments/indices qui classent Istanbul, Souvenirs d'une ville dans le genre autobiographique tel qu'il est définit par Philippe Lejeune tout en se questionnant sur les aspects qui pourraient l'en éloigner, et d'autre part essayer de donner des exemples illustrant les moyens par lesquels les souvenirs se cristallisent dans ce triangle remémoratif.

\section{Le titre et ses indices autobiographiques}

Il serait intéressant de commencer par une analyse du titre dont nous rappelons la version originale: Istanbul, Hatıralar ve Şehir. Traduit vers le français (2007) après la traduction anglaise (2005), le titre de la version française Istanbul, Souvenirs d'une ville reprend sur le plan de la syntaxe et de la grammaire le même titre que celui que nous retrouvons dans la première édition

\footnotetext{
${ }^{2}$ Nos références porteront sur la traduction française.
} 
de la traduction vers l'anglais: Istanbul, Memories of a City ${ }^{3}$. Le sous-titre proposé en français est construit à partir d'un complément du nom et ce complément du mot "souvenirs » est introduit par l'article indéfini "une » comme c'est d'ailleurs le cas pour la première édition publiée en anglais. Le titre principal « Istanbul » montre qu'il s'agit d'un récit concernant la ville en question et le sous titre en turc «Hatıralar ve Şehir » renvoient à deux aspects différents, aspects qui d'ailleurs sont séparés par la conjonction de coordination «ve» (et) qui a une valeur additive. Quant au mot « şehir » (ville), celui-ci est introduit par un article défini, du moins l'article indéfini « bir » (un/une) n'est pas utilisé: il y a « les » ou « des » souvenirs et il y a « la » ville. Le titre en turc a donc une valeur sémantique différente du titre en français qui semble évoquer simplement les « souvenirs » de cette ville sans faire référence à la ville elle même. En outre, le titre en anglais Istanbul, Memories and the City, utilisé après la première édition semble se rapprocher de la valeur sémantique du titre original. Ainsi pour revenir dans le contexte du biographique, nous pouvons dire que le titre original fait référence non seulement au genre autobiographique (souvenirs d'Orhan Pamuk), mais aussi à la description d'une ville que l'on pourrait qualifier de "chronique de la ville » au fil de la lecture. Ce n'est évidemment qu'au fil de la lecture que l'on s'aperçoit que l'on ne peut imaginer l'histoire de Pamuk sans l'histoire d'Istanbul. En effet, l'histoire d'Istanbul accompagne l'histoire de l'auteur si bien que ces deux histoires qui portent en elles le même état d'âme se fondent même souvent en une seule image, voire en une seule personne. Ainsi, Istanbul considérée/décrite dans le contexte d'une capitale d'un empire déchu, vivant les années les plus miséreuses de ses deux mille ans d'histoire, correspond très bien au sous-titre proposé dans la version française. Istanbul, Souvenirs d'une ville, c'est aussi bien l'Istanbul actuelle (deuxième personnage principal du livre) qui se souvient de ce qu'elle a été que Pamuk, indissociable d'Istanbul, qui se souvient de ce qu'était sa ville un siècle auparavant, grâce aux gravures du dessinateur français Melling auquel un chapitre est consacré et grâce aux récits des écrivains voyageurs français, notamment Nerval, Gautier et Flaubert, auxquels trois chapitres sont consacrés. Dans cette optique, nous pouvons dire que le mot « souvenirs » du sous-titre renvoie non seulement à Istanbul/Pamuk mais aussi à tous les souvenirs des dessinateurs et écrivains qui ont produit des œuvres sur Istanbul.

En terminant cette partie concernant le titre de l'œuvre et en guise de première conclusion nous pouvons dire que les titres - l'original ainsi que le titre traduit qui nous semble malgré tout bien approprié si nous le considérons par rapport à l'intégralité de l'œuvre- portent en eux non seulement des références autobiographiques, mais aussi des indices concernant une biographie/chronique de la ville.

\footnotetext{
${ }^{3}$ Les recherches effectuées sur internet affichent aussi, pour les éditions qui ont suivi, l'utilisation du titre: Istanbul, Memories and the City.
} 


\section{Autour des indices (auto)biographiques}

Pour mieux se prêter à une analyse du biographique, il va sans dire qu'il est indispensable de questionner l'écriture elle-même. Même si l'auteur annonce son objectif, il peut toutefois s'en éloigner, et l'analyse de l'écriture permet de créer le lien qui existe entre la réalité présentée dans l'œuvre en question, la réalité vécue et la part de «fiction », si celle-ci est présente dans l'œuvre. Avant de questionner l'incipit d'Istanbul, Souvenirs d'une ville, rappelons deux œuvres relevant du biographique pour mieux saisir ce que nous rechercherons dans les premières pages de l'œuvre de Pamuk.

Le théologien Saint Augustin qui a vécu au Vème siècle, est reconnu avec ses Confessions dans lequel il dialogue avec le "Créateur » comme le premier écrivain à avoir entrepris une autobiographie correspondant à la définition que Philippe Lejeune en fait dans Le Pacte autobiographique: "Récit rétrospectif en prose qu'une personne réelle fait de sa propre existence, lorsqu'elle met l'accent sur sa vie individuelle, en particulier sur l'histoire de sa personnalité » (1975, p. 14). Cependant, il faudra attendre Les Confessions de Rousseau pour témoigner d'un genre qui va loin dans la peinture de soi et qui correspond à l'autobiographie en tant que genre littéraire. En effet, dans le préambule des Confessions Rousseau annonce son objectif et met en place un pacte avec son lecteur: « Je forme une entreprise qui n'eut jamais d'exemple et dont l'exécution n'aura point d'imitateur (...) J'ai dit le bien et le mal avec la même franchise »(1961, p. 5). Qu'en est-il pour Istanbul, Souvenirs d'une ville? Pamuk met-il en place un pacte avec ses lecteurs ? En quoi correspond-il au genre biographique?

S'agissant d'un récit rétrospectif en prose que l'auteur fait de sa propre existence (Lejeune, 1975) sans qu'il y ait d'ambigüité quant à la triple identité auteur-narrateur et personnage (Clerc, 2001, p. 21), Istanbul, Souvenirs d'une ville peut donc être classé parmi les différentes formes de l'autobiographie. Cependant, étant donné que l'auteur raconte des fragments de sa vie, en relation à l'Histoire dont il a été témoin et à la ville qui l'a vu naître, l'œuvre doit être considérée comme une des formes « voisines » de l'autobiographie: les mémoires. Même si la plupart des mémoires correspondent à la définition que Lejeune fait de l'autobiographie, il nous faut rappeler que les mémoires « tendent à relativiser la notion de personne en mettant l'accent sur l'histoire " (Clerc, 2001, p. 60). Pour le mémorialiste l'objectif principal est donc de créer un lien entre l'histoire, la société et son vécu; objectif qui correspond assez bien à celui d'Istanbul, Souvenirs d'une ville.

Il serait intéressant de s'attarder sur le pacte autobiographique qu'Orhan Pamuk met en place avec le lecteur en clôturant le premier chapitre. Il y définit assez clairement son objectif, qui n'est pas de faire un conte, mais de faire un récit dans lequel il insiste sur l'identité entre l'auteur, le narrateur et le personnage:

Je trouve que présenter son histoire sous une forme de conte ne serait pas crédible, dans la mesure où elle nous préparerait à une deuxième vie où, une fois le conte terminé, comme si l'on venait de sortir d'un rêve, les choses paraitraient plus vraies et plus précises. En effet, la deuxième vie que mes semblables et moi- 
même peuvent mener n'est pas autre chose que le livre qu'ils tiennent entre les mains. Cela aussi dépend de ton attention, ô lecteur. Il faut que je fasse preuve de franchise à ton égard, et toi de sollicitude envers moi (2007, p. 20).

Cependant, nous remarquons à maintes reprises que l'auteur prend parfois du recul par rapport à la narration qu'il nous livre à la première personne, voire par rapport à la triple identité. En effet dans le troisième chapitre intitulé " moi » comme dans plusieurs autres passages, l'utilisation de la troisième personne du singulier est assez fréquente chez Orhan Pamuk « adulte » qui raconte des fragments d'histoires concernant le «petit » Orhan effrayé devant l'image du géant dans la gueule duquel tombe la petite souris Miki:

Orhan se mettait alors à pleurer à grand sanglots. Aujourd'hui encore, la toile de Goya exposée au musée du Padro intitulée Saturne en train de dévorer un de ses enfants -que je vois comme la représentation d'un petit homme qu'un géant arrache du sol pour le porter à sa bouche- continue à me glacer le sang $(2007, \mathrm{p}$. 39).

Dans le contexte des mémoires, et comme nous pouvons le remarquer dans le passage ci-dessus, « la troisième personne a un rôle objectivant puisqu'elle signale de fait un écart entre le narrateur et le personnage » (Clerc, 2001, p. 61). Nous ne pouvons d'ailleurs pas dire que cela entraîne une ambigüité au niveau de la triple identité auteur-narrateur-personnage principal car nous savons qu'il y a une identité entre le « je » et le « il ». En effet, dans l'incipit, l'auteur raconte la rencontre entre les deux Orhan Pamuk, c'est-à-dire celui qui figure sur la photo se trouvant chez sa tante et vivant dans une maison autre que celui vivant dans l'immeuble Pamuk: "L'idée qu'un autre Orhan vivait dans une autre maison quelque part à Istanbul ne m'a jamais abandonné » rappelle le narrateur à plusieurs reprises (p. 16, 46, 47) pour évoquer un monde qui se construit parallèlement au sien. Effectivement tout au long de l'œuvre, l'auteur porte un regard vers ce petit Orhan qu'il reconstitue à la troisième personne du singulier sous le regard et l'écriture de l'Orhan Pamuk adulte. Ce dernier recourt souvent à cette forme de narration pour s'évader de son monde et pour introduire le monde imaginaire que le petit Orhan s'était construit. Cependant, pendant ces va-et-vient rétrospectifs qui s'étendent sur différentes périodes de la vie de l'auteur, Orhan Pamuk dialogue avec le lecteur avec qui il a passé un pacte. Ainsi, racontant les années pendant lesquelles il est attiré par le dessin, il ne manque pas de prendre du recul par rapport à son texte pour faire une autoanalyse. Il en profite pour confier au lecteur qu'il éprouve des difficultés lors de la rédaction de son récit intime :

Pourquoi éprouvais-je un tel plaisir à dessiner? De quoi ce plaisir était-il fait? Ici, votre mémorialiste va quelque peu éloigner son récit de la conscience du petit enfant et se rapprocher de celle de l'écrivain de cinquante ans, qui croit parvenir à se raconter en s'efforçant de comprendre ce petit enfant (2007, p. 220).

Le passage ci-dessus nous montre qu'il est effectivement difficile de se livrer à un récit de vie, mais il fait preuve aussi de la sincérité de l'auteur, un des aspects de l'autobiographie. Sincérité que nous retrouvons dans le chapitre trente deux lorsqu'il raconte les querelles et la violence des bagarres avec son 
grand frère qui, selon ce dernier et sa mère ne s'étaient jamais passés et seraient par conséquent le fruit d'une imagination essayant de s'inventer un passé original. Pamuk ne manque pas d'avertir le lecteur:

le lecteur qui lit actuellement ces lignes doit garder dans un coin de sa tête que je n'ai toujours pas pu me libérer de l'emprise de mon imagination (...) pour un écrivain qui écrit ses souvenirs, ce qui importe, ce n'est pas la réalité du passé, mais sa symétrie (...) C'est pourquoi le lecteur qui s'est rendu compte que parler de moi revenait à parler d'Istanbul, et vice versa, a déjà dû comprendre (...) que le récit des bagarres (...) sert en fait à préparer le terrain pour d'autres choses (2007, p. 428-429).

Après la lecture du passage cité, on ne peut se retenir de penser à Philippe Lejeune qui mettait en garde les lecteurs en soulignant le fait que « l'autobiographie ne dit pas vrai, elle dit qu'elle dit vrai » (1975, p. 15).

Les va-et-vient entre les différentes périodes du passé et le présent de l'auteur de cinquante ans semblent bien plus difficiles qu'il ne l'y parait, surtout qu'il s'agit aussi pour cet auteur de reconstituer en parallèle à ses souvenirs une biographie/chronique de la ville Istanbul dont la beauté réside dans la tristesse, dira-t-on, pour se rapprocher de l'épigraphe d'Ahmet Rasim: La beauté d'un paysage réside dans sa tristesse.

\section{Les embrayeurs mémoriels}

Essayons maintenant de créer un parallèle entre les indices de la biographie/chronique concernant la ville Istanbul et les indices autobiographiques concernant le personnage principal en nous appuyant non seulement sur des épisodes de l'histoire mais aussi sur différentes figures littéraires et artistiques que nous pouvons considérer comme des embrayeurs agissant sur la complexité du phénomène mémoriel et facilitant la narration concernant les souvenirs.

Sans soucis de chronologie, le récit relate et remémore, principalement, plusieurs étapes différentes des vingt deux premières années de la vie de l'auteur en parallèle avec le récit de la ville Istanbul qui, parfois, devient le deuxième personnage principal de l'œuvre. Comme il le rappelle lui-même par moments dans le livre, Pamuk est né à une période où l'Empire ottoman n'existe plus et où Istanbul plongée dans la tristesse « vivait les jours les plus faibles, les plus misérables, les plus sombres et les moins glorieuses de ses deux mille ans d'histoire » $(2007$, p. 18). Il va sans dire que le sentiment de tristesse qui règne sur la ville est le fil conducteur du récit. Pamuk affirme dans l'incipit «Istanbul, la ville où je suis né et où j'ai passé toute ma vie (...) fait partie du destin (...) ce livre est à propos de ce destin $(2007$, p. 19) ou encore « parler de moi revient à parler d'Istanbul et vice-versa » (2007, p. 429) dira-t-il plus loin. Pamuk intériorise donc solidement cette union à partir de laquelle il crée un lien entre Istanbul, sa vie et son entourage familial. Ainsi, les moments où ses parents se disputaient sans cesse, les années pendant lesquelles les faillites de son père et de son oncle se répétaient, voire ces moments provoquant «par endroits des fissures qui ont rapidement effrité et appauvri la famille » sont associés au «sentiment de défaite, de perte et de tristesse dont Istanbul avait 
hérité suite à la chute de l'Empire ottoman » (2007, p. 35). Les photos en noir et blanc de sa famille et celles d'Istanbul provenant pour la plupart de l'album Istanbul Perdu de Ara Güler, qui sont joints au récit et qui font référence au poids des ans et à la tristesse de la ville contribuent à rendre encore plus triste l'atmosphère qui règne sur la ville. De même, les gravures en noir et blanc d'Antoine Ignace Melling ou encore les dessins au fusain réalisés par Le Corbusier sont des supports qui reflètent non seulement l'esprit noir et blanc d'Istanbul mais aussi, comme le souligne Sarga Moussa, des supports sur lesquels Pamuk s'appuie pour composer son kaléidoscope mémoriel et cristalliser son souvenir ${ }^{4}$. Ainsi les paysages qui figurent sur les quelques gravures parmi les quarante huit gravures du Voyage pittoresque de Constantinople et des rives $d u$ Bosphore que Pamuk a joints au texte, permettent à l'auteur d'avoir « une exacte idée de ce qu'était l'impeccable Istanbul ottoman dans le passé » (2007, p. 99), mais c'est aussi à partir de la beauté de ces paysages disparus que Pamuk peut relater sa tristesse et celle de «sa ville». Et pourtant, tristesse et bonheur se rencontrent confie le mémorialiste en notant que c'est grâce à ces gravures que ses souvenirs se cristallisent. En effet, Pamuk retrouve sur ces gravures bien détaillées beaucoup de points communs aux années de son enfance : les vendeurs de pastèques, les vendeurs de rue au visage marqué par une expression de lassitude, le montreur d'ours sur la colline de Kandilli et son compagnon jouant du tef, le vendeur de simit posés sur un trépied, etc. (2007, p. 110-111).Tout comme Melling qui embraye les souvenirs et génère l'écriture de l'auteur, des écrivains français tels Nerval, Baudelaire, Gautier, Flaubert, Lamartine ou encore Pierre Loti, tous des écrivains qui ont séjourné à Istanbul et qui ont décrit la ville, alimentent le récit autobiographique de Pamuk. Les écrivains français tels Montaigne, Balzac (son Rastignac), Gide, etc., sont aussi évoqués dans le récit, mais dans une perspective qui vise à faire des comparaisons pour éclaircir certaines notions ou à soutenir la prise de position de l'auteur sur certains sujets. A titre d'exemple, en parlant du « hüzün » Pamuk signale que Montaigne maitrisait la tristesse en vivant « isolé avec ses livres alors que Istanbul vit le hüzün en tant que grande ville où tout le monde concourt à affirmer ce sentiment » (2007, p. 158). Quant au Rastignac de Balzac, il est le modèle d'une personne qui affirme sa volonté d'être un individu face à la société; ce que les habitants d'Istanbul ne peuvent affirmer.

Pour revenir aux auteurs qui se sont trouvés à Istanbul et plus particulièrement à Nerval, Baudelaire, Gautier et Flaubert, Pamuk évoque les représentations, les décors, voire les récits que ces derniers ont faits du paysage pittoresque et humain d'Istanbul. Effectivement, les flâneries « littéraires que ces écrivains ont faites dans Istanbul et les descriptions des aspects exotiques qu'ils en ont faites représentent une passerelle qui permet à l'auteur de se souvenir de l'Istanbul oubliée, voire disparue de son enfance. Les deux Istanbul, celle vue et décrite

\footnotetext{
4 Orhan Pamuk lecteur des écrivains voyageurs français à Constantinople au XIXe siècle <http://halshs.archives-ouvertes.fr/halshs-00415857>, consulté le 18 novembre 2013.
} 
par les écrivains voyageurs français et celle vue par « le petit Orhan » ont en effet beaucoup de points communs. Et, ce n'est pas par pur plaisir littéraire, nous semble-t-il, que Pamuk a associé à ce récit autobiographique des fragments de biographies de ces écrivains français atteints du «mal du siècle ». Ils ont tous un point commun qui, à la fois, les différencient de Pamuk: la mélancolie chez les écrivains français et le hüzün chez Pamuk. Si comme Baudelaire, Gautier, dans son livre intitulé Constantinople (1853), utilisait le terme mélancolie dans un sens positif pour « qualifier certains paysages qu'il trouvait par trop mélancoliques » (2007, p. 142), Nerval, lui, entreprend son périple en Orient pour oublier sa propre mélancolie due notamment aux frustrations et souffrances entraînées par sa liaison avec Jenny Colon. C'est dans cet état d'âme qu'il s'abandonne « aux rêves colorés de l'Orient stéréotypé des occidentaux » (2007, p. 155). Chez Pamuk par contre, la source du sentiment du hüzün qui l'envahit et qui s'abat sur Istanbul et ses habitants provient d'une part « des conséquences de l'histoire et de l'effondrement de l'Empire ottoman et de la façon dont cette histoire se reflète dans les «beaux» paysages et les habitants de la ville » (2007, p. 139) note l'auteur.

Quant aux descriptions que Gautier fait de l'Istanbul labyrinthique, de ses obscures maisons en bois à l'état de ruine, de ses murs en pierre, de ses rues désertes, de ses fontaines hors d'usage, des cyprès signalant les cimetières, etc. , celles-ci alimentent la mémoire de Pamuk en rappelant les paysages qu'il a pu voir avec son père en se promenant en voiture. Toutes ces descriptions qui procurent un sentiment de mélancolie dans un sens positif chez les écrivains français provoquent une tristesse très profonde chez l'auteur qui conclut: « le sentiment fondamental qui règne sur Istanbul et s'est propagé dans les environs de la ville ces cent cinquante dernières années (1850-2000) est incontestablement de la tristesse » $(2007$, p. 45).

\section{Conclusion}

Si nous passons sous silence les quelques dialogues que nous rencontrons dans les paragraphes trente cinq et trente sept, nous pouvons dire qu'Istanbul, Souvenirs d'une ville, structuré sous forme de monologue intérieur est autant un récit rétrospectif accompagné de commentaires et de jugements apportés par l'auteur qu'un portrait ou une « biographie » de la ville Istanbul dont le destin rejoint celui de l'écrivain. C'est d'ailleurs pendant une période triste et chaotique due au départ pour la Suisse de Rose Noire, son premier amour et peut-être premier modèle à avoir posé et, suite à ses interminables et fatigantes discussions avec sa mère, s'opposant à son désir de devenir peintre, que Pamuk, alors âgé de 22 ans, décide d'écrire quelque chose sur l'Istanbul triste et miséreuse qu'il associe/identifie à son état d'âme, voire à son destin: « L'envie subite de rentrer en courant à la maison et d'écrire quelque chose pour fixer ces images, cette âme obscure, ce désordre chaotique, cet aspect mystérieux et fatigué de la ville s'emparait de moi » (2007, p. 528). Istanbul, Souvenirs d'une ville est ainsi, autant le journal de la vie d'Istanbul et son cahier de souvenirs que les mémoires d'un écrivain qui ne peut échapper au courant de l'écriture intime très en vogue depuis les quelques dernières décennies. 


\section{KAYNAKÇA}

Clerc, T. (2001). Les écrits personnels. Paris: Collections Ancrages, Hachette.

Lejeune, P. (1975). Le Pacte autobiographique. Paris: Éditions Seuil.

Maillard, M. (2001). L'autobiographie et la biographie, Genres et Mouvements. Paris: Collection Balises, Nathan.

Moussa, S. (2009), Orhan Pamuk lecteur des écrivains voyageurs français à Constantinople au XIXe siècle, <http://halshs.archives-ouvertes.fr/halshs00415857>, consulté le 18 novembre 2013.

Pamuk, O. (2003). İstanbul, Hatıralar ve Şehir. İstanbul: Yapı Kredi Yayınları.

Pamuk, O. (2007). Istanbul, Souvenirs d'une ville. (Traduit du turc par Savaş Demirel, Valérie Gay-Aksoy et Jean-Françpis Pérouse). Paris: Éditions Gallimard.

Rousseau, J.-J. (1961). Les Confessions. Paris: «Bibliothèque de la Pléiade», Gallimard.

Augustin, S. (1964). Les Confessions. Paris: GF Flammarion.

\section{REMINISCENT TRIAD: ISTANBUL - YOUNG PAMUK - FRENCH ARTISTS AND AUTHORS}

Abstract: Orhan Pamuk's work entitled İstanbul, Hattralar ve Şehir (2003) is an autobiographical account of the first twenty two-years that the author spent in Istanbul and also a reflection of the views and observations of the author about the period which he lived in. In addition to this, as can be understood from the title, this work is also be considered as an autobiography of the city of Istanbul itself. In this context, the author brings together the story of a child, a teenager and a university student- rather the author himself- with Istanbul. The narrator-author Orhan Pamuk, as he recites the life of the young Orhan which overlaps with Istanbul in a separate section, refers to the engravings of French traveler, architect and painter Antoine Ignace Melling (1763-1831) about Istanbul; and in other sections he refers to the writings about Istanbul of the19th century French authors Théophile Gautier, Gérard de Nerval and Gustave Flaubert as a subtext. The fact that Pamuk corresponds with the authors is a way to enliven or visualize his memories that he is trying to pen as he urges us to read their works from a different perspective. The subtexts used in the autobiography and the memories resurrected through these make the reader doubt the reality of the autobiography, but one has to believe in the pacte autobiographique that the author establishes with himself to be able to relay the whole truth. After a comparison of the title in Turkish (Istanbul, Hatıralar ve Sehir) with the title in French (Istanbul, Souvenirs d'une ville) within the scope of biographical and autobiographical metaphors, we will discuss how far this work reflects (Le Pacte autobiographique) that initial definition by Philippe Lejeune in 1975 , how far there is an effort to relay the reality to the readers, and how far the author relayed this. 
Keywords: Orhan Pamuk, Istanbul, Souvenirs D'une Ville, Autobiographie, Récit Intime, Récit Rétrospectif, Embrayeurs Biographiques, Rencontres Biographiques, Biographie Urbaine, Melling, Baudelaire, Flaubert, Gautier, Nerval. 